\title{
MODEL OF A DIPOLE WITH ATOMIC STRUCTURE
}

\author{
D.O. Vasylenko ${ }^{1}$, V.S. Sydorenko ${ }^{1}$, P.O. Kravchuk ${ }^{1}$, V.I. Grygoruk ${ }^{1}$, V.I. Kanevskii ${ }^{2}$ \\ ${ }^{I}$ Taras Shevchenko National University of Kyiv \\ Volodymyrska Str., 64/13, Kyiv, Ukraine, 01601 \\ ${ }^{2}$ Chuiko Institute of Surface Chemistry of National Academy of Sciences of Ukraine \\ General Naumov Str., 17, Kyiv, Ukraine, 03164 \\ e-mail:vvda7@ukr.net \\ Received December 27, 2017
}

In this paper, we propose a model of a dipole with an atomic structure instead of the standard dipole model with point unlike charges and the Hertzian dipole model, which have significant drawbacks. The equations of the Hertzian dipole and the standard model operate from a distance much larger than size of the dipole, and the quasistatic Coulomb and Biot-Savart fields are the essence of the reactive near field, its own fields with a phase shift $\Delta \varphi_{E, H}=\pi / 2$, which have no restrictions on the distances to a dipole, since they are directly related to charges and their motion - currents. In the framework of the proposed dipole model, we described the physical mechanisms for the formation of near and far fields of an oscillating dipole, which are based on the use of the Coulomb and BiotSavart fields, the quasistatic lines of force of their electric charge fields $\boldsymbol{E}$, and the magnetic fields of the currents $\boldsymbol{H}$ for the analysis of energy fluxes: reactive $\boldsymbol{S}_{r}$ at $\Delta \varphi_{E, \boldsymbol{H}}=\pi / 2$ and active $\boldsymbol{S}_{a}$ at $\Delta \varphi_{E, \boldsymbol{H}}=0$ alike.

KEY WORDS: dipole, near field, far field, decompensation, oscillation

\section{МОДЕЛЬ ДИПОЛЯ ЗІ СТРУКТУРОЮ АТОМА \\ Д.О. Василенко ${ }^{1}$, В.С. Сидоренко ${ }^{1}$, П.О. Кравчук ${ }^{1}$, В.І. Григорук ${ }^{1}$, В.І. Кансвський ${ }^{2}$ \\ ${ }^{1}$ Київський національний університет імені Тараса Шевченка вул. Володимирська, 64/13, Київ, Україна, 01601 \\ ${ }^{2}$ Інститут хімії поверхні ім. О.О. Чуйка Національної академії наук Украӥни вул. Генерала Наумова, 17, Київ, Украӥна, 03164}

В роботі запропонована модель диполя зі структурою атома замість стандартної моделі диполя з точковими різнойменними зарядами та моделі диполя Герца, які мають істотні недоліки. Рівняння диполя Герца і стандартної моделі виконуються для відстані, що значно перевищує розмір самого диполя, а квазістатичні поля Кулона і Біо-Савара $є$ суттю реактивного ближнього поля, його власними полями зі зсувом фаз $\Delta \varphi_{E, H}=\pi / 2$, які не мають обмежень на відстань до диполя, оскільки безпосередньо зв'язані з зарядами та їхнім рухом - струмами. В рамках запропонованої моделі диполя нами були описані фізичні механізми формування ближніх і дальніх полів осцилюючого диполя, які грунтуються на використанні полів Кулона та Біо-Савара, квазістатичних силових ліній їх електричних зарядових полів $\boldsymbol{E}$ і магнітних полів струмів $\boldsymbol{H}$ для аналізу потоків енергії: як реактивних $\boldsymbol{S}_{r}$ при $\Delta \varphi_{\boldsymbol{E}, \boldsymbol{H}}=\pi / 2$, так і активних $\boldsymbol{S}_{a}$ при $\Delta \varphi_{\boldsymbol{E}, \boldsymbol{H}}=0$.

КЛЮЧОВІ СЛОВА: диполь, ближне поле, дальне поле, розкомпенсація, осциляція

\author{
МОДЕЛЬ ДИПОЛЯ СО СТРУКТУРОЙ АТОМА \\ Д.А. Василенко ${ }^{1}$, В.С. Сидоренко ${ }^{1}$, П.А. Кравчук ${ }^{1}$ В.И. Григорук ${ }^{1}$, В.И. Каневский ${ }^{2}$ \\ ${ }^{1}$ Киевский национальный университет имени Тараса Шевченко \\ ул. Владимирская, 64/13, Киев, Украина, 01601 \\ ${ }^{2}$ Институт химии поверхности им. А.А. Чуйко Нацчиональной академии наук Украины \\ ул. Генерала Наумова, 17, Киев, Украина, 03164
}

В работе предложена модель диполя со структурой атома вместо стандартной модели диполя с точечными разноименными зарядами и модели диполя Герца, которые имею существенные недостатки. Уравнения диполя Герца и стандартной модели работают от расстояния, намного превышающего размер самого диполя, а квазистатические поля Кулона и Био-Савара являются сутью реактивного ближнего поля, его собственными полями со сдвигом фаз $\Delta \varphi_{E, H}=\pi / 2$, которые не имеют ограничений на расстояния к диполю, поскольку непосредственно связаны с зарядами и их движением - токами. В рамках предложенной модели диполя нами были описаны физические механизмы формирования ближних и дальних полей осциллирующего диполя, которые основаны на использовании полей Кулона и Био-Савара, квазистатических силовых линий их электрических зарядовых полей $\boldsymbol{E}$ и магнитных полей токов $\boldsymbol{H}$ для анализа потоков энергии: как реактивных $\boldsymbol{S}_{r}$ при $\Delta \varphi_{E, \boldsymbol{H}}=\pi / 2$, так и активных $\boldsymbol{S}_{a}$ при $\Delta \varphi_{\boldsymbol{E}, \boldsymbol{H}}=0$.

КЛЮЧЕВЫЕ СЛОВА: диполь, ближнее поле, дальнее поле, раскомпенсация, осцилляция

В классической электродинамике есть модель диполя Герца [1-4]. Это бесконечно малый бесструктурный элемент тока - фактически «чёрный ящик». В модели Герца нет реальных внутридипольных векторов $\boldsymbol{E}, \boldsymbol{H}$ и $\boldsymbol{S}$ [1], что не позволяет рассмотреть физические механизмы формирования реактивных и активных потоков энергии, соответственно ближнего и дальнего полей. В последнее время все больше и больше исследований: как теоретических $[5,6]$, так и экспериментальных $[7,8]$, показывают принципиальное отличие физических процессов, происходящих в ближнем и дальнем полях. В диполе Герца нет понятия межзарядовой силы $\boldsymbol{F}$, a следовательно и механизма установления равновесия амплитуды колебаний $l_{0}$ дипольных зарядов под действием внешнего индуцирующего поля $\boldsymbol{E}_{0}$. 
В стандартной модели диполем является система, состоящая из двух точечных зарядов $+q$ и $-q$, расположенных на расстоянии $l_{0}$ друг от друга, причём $r \gg l_{0}$ (где $r$ - расстояние от диполя к точке наблюдения) $[9,10]$. Точечность зарядов этой стандартной модели должна допускать использование закона Кулона: $F \sim\left(q_{1} \cdot q_{2}\right) / l^{2}$. Но попытка применения закона Кулона к зарядам атомного диполя приводит при $l \rightarrow 0$ к сингулярности в нулевой точке (где заряды совмещаются и диполь схлопывается), так как при этом $\mathrm{F} \rightarrow \infty$. И никакое внешнее поле (с конечной силой) не сможет развести заряды и поляризовать атом.

Известно, что при приложении внешнего поля атомы поляризуются и их дипольные моменты осциллируют с участием внутренних межзарядовых сил. Но это не силы закона Кулона - внутри атомного диполя закон Кулона не работает. Очевидно, что необходима новая модель диполя и другая формула для внутридипольной силы между зарядами осциллирующего диполя. Необходимо принципиально изменить сам подход к пониманию процессов, происходящих при осцилляции диполя.

Цель работы состоит в разработке новой модели диполя, более приближенной к реальности, нежели модель диполя Герца или стандартная модель с разнесенными точечными зарядами, а также описание преобразования реактивного ближнего поля в активное поле излучение с использованием предложенной модели.

\section{ПОЛОЖЕНИЯ МОДЕЛИ}

В отличие от бесструктурной модели диполя Герца или стандартной модели с точечными зарядами мы предложили использовать для модели диполя реальную зарядовую структуру атома, в которой положительный заряд ядра всегда находится внутри электронной оболочки, и назвали её «моделью диполя со структурой атома». Эта модель функционирует для любых атомов и молекул, так как все они поляризуются во внешнем поле, образуя осциллирующие диполи. Предложенная атомная модель диполя на основе квазистатической электродинамики работает вне резонансов.

Собственные поля зарядов ядра и электронов атома порождаются процессами, которые имеют место быть внутри этих частиц. Данные процессы в статье не рассматриваются, поскольку выходят за рамки классической электродинамики. Их проявления видны только во внешних силовых полях зарядов.

Поскольку электронные орбитали центрально симметричны [11], то вместо пространственно распределенных по ним зарядов электронов мы ввели в предложенную модель диполя понятие «эквивалентного отрицательного заряда» $(-q)$, равного сумме зарядов всех электронов атома и расположенного в центре симметрии его электронных орбиталей. Это не реальный заряд, а его эквивалентная модель, облегчающая описание физических механизмов, происходящих при осцилляции реального диполя со структурой атома.

Центр положительного заряда $+q$ (ядра) всегда находится внутри электронной оболочки (рис. 1), таким образом, поля зарядов $+q$ и $-q$ всегда перекрываются и частично компенсируют друг друга. Без внешнего поля $\left(\boldsymbol{E}_{0}=0\right)$ центры зарядов $+q$ и $-q$ совмещены, компенсация зарядовых полей полная и атом неполяризован (рис. 1, a).

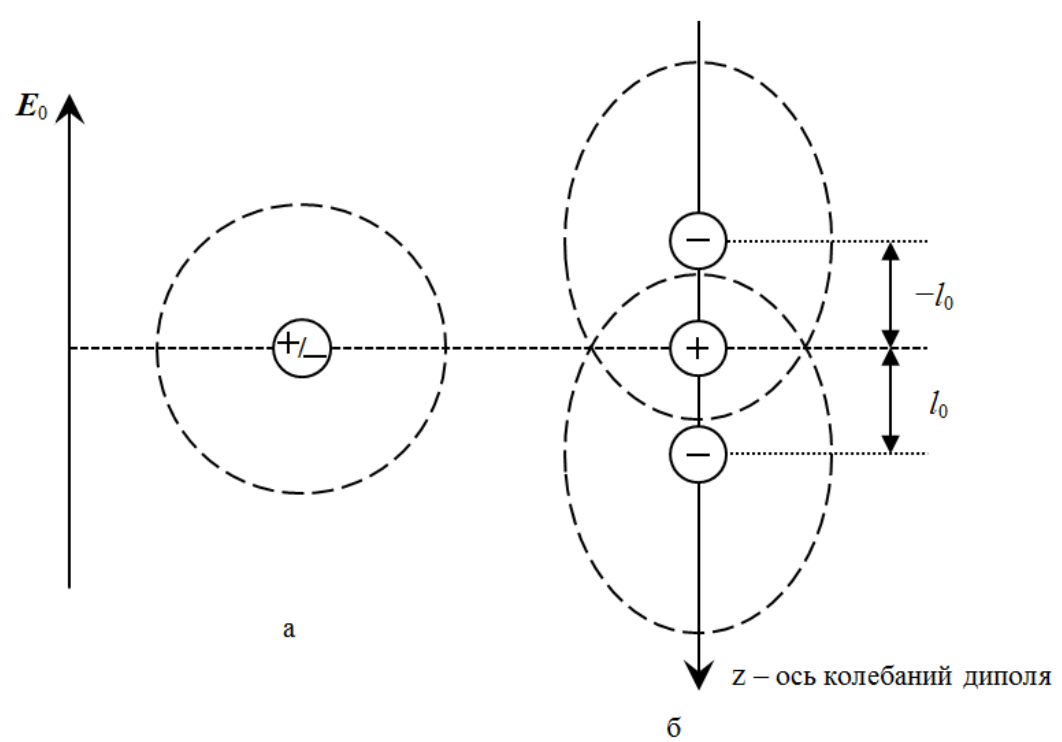

Рис. 1. Поляризация атома во внешнем электрическом поле

(a - атом неполяризован, б - атом поляризован и осциллирует). Разнонаправленность осей $\boldsymbol{E}_{0}$ и z указывает на смещение электронной оболочки против внешнего поля, «+» - положительный заряд ядра атома, «-» - эквивалентный отрицательный заряд электронного облака. Пунктиром обозначено электронное облако

Во внешнем поле $\left(\boldsymbol{E}_{0} \neq 0\right)$ диполь осциллирует (рис. 1, б). В процессе осцилляции происходит 
интерференция полей двух зарядов, одинаковых по величине, но противоположных по знаку. Ядро на порядки тяжелее электронов и поэтому практически неподвижно. Центры разноименных зарядов то отдаляются друг от друга (расходятся), то сближаются (сходятся). При этом они компенсируют то меньшую, то бо́льшую часть собственных квазистатических полей друг друга и их силовых линий. Сами поля остаются при своих зарядах, но интерференция полей разноименных зарядов уменьшает их внешнее силовое проявление, изменяя плотность силовых линий. Значительная часть силовых линий обоих зарядов находится в связанном состоянии взаимной компенсации и не проявляет себя в силовом взаимодействии. Это можно назвать интерференцией квазистатических полей. Таким образом, ближнее поле - это собственные квазистатические поля (силовые линии) Кулона и Био-Савара двух колеблющихся зарядов диполя, поля которых интерферируют при его осцилляции. Но внешне это выглядит как пульсация электромагнитного поля, реактивного по сдвигам фаз и знакопеременности потоков энергии.

Заметим, что электронная оболочка всегда содержит внутри себя не только эквивалентный отрицательный заряд, но и положительный заряд ядра, то есть фактически весь диполь. В данной модели осью диполя является прямая, проходящая через центр положительного заряда ядра и центр эквивалентного отрицательного заряда электронного облака атома.

При приложении внешнего поля электронная оболочка смещается (вытягивается) относительно ядра против поля и центры зарядов $+q$ и $-q$ расходятся (рис. 2, а). Атом поляризуется за счёт частичной раскомпенсации перекрывающихся полей расходящихся разноименных зарядов.

При изменении знака внешнего поля, направление смещения электронной оболочки изменяется на противоположное (рис. 2, б; 1, б). Силовые линии электрического поля диполя, согласно электростатике, начинаются на положительном заряде и заканчиваются на отрицательном. Они всегда противоположны внешнему полю $\boldsymbol{E}_{0}$ и поэтому частично компенсируют его, чем и объясняется уменьшение амплитуды волны в диэлектрике в $\varepsilon$ раз (где $\varepsilon-$ диэлектрическая проницаемость).

В процессе смещения эквивалентного отрицательного заряда электронной оболочки возникает ток, который по закону Био-Савара генерирует вокруг линии тока (оси диполя $l$ ) замкнутые силовые линии тороидального квазистатического магнитного поля. Направление тока противоположно смещению электронной оболочки, то есть совпадает с направлением внешнего поля.

Важным элементом предложенной атомной модели является то, что в процессе поляризации, то есть при изменении плеча диполя $l$, меняется величина раскомпенсации полей (этого нет в стандартной модели и модели диполя Герца). В стандартной модели диполя раскомпенсация полей постоянная, что не соответствует действительности. А диполь Герца - бесструктурный, что не позволяет рассмотреть явление раскомпенсации в принципе.

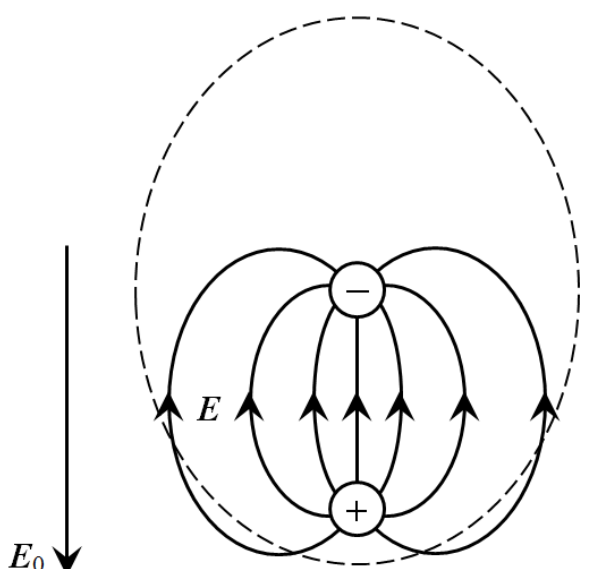

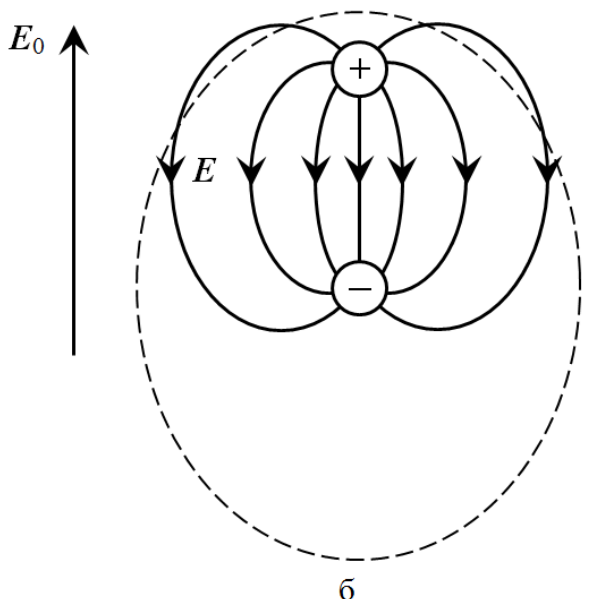

Рис. 2. Атомный диполь во внешнем электрическом поле при разных направлениях вектора напряженности (а и б). «+» - положительный заряд ядра атома, «-» - эквивалентный отрицательный заряд электронного облака.

Пунктиром обозначено электронное облако.

С увеличением внешнего поля центр электронной оболочки всё дальше смещается от положительного ядра, что приводит к еще большей раскомпенсации перекрывающихся полей зарядов $+q$ и $-q$. Таким образом, при изменении внешнего поля дипольный момент $\boldsymbol{p}=q \boldsymbol{l}$ меняется за счёт эффекта раскомпенсации.

Очевидно, что для описания сил притяжения между противоположными по знаку зарядами в процессе частичной раскомпенсации их полей под действием внешнего поляризующего поля вместо формулы закона Кулона необходима другая формула, которая учитывает изменение величины раскомпенсации полей зарядов с изменением плеча диполя $l$ (рис. 3).

Для исследуемой модели диполя нами предложена функциональная формула дипольной силы, возникающей между разноименными зарядами в процессе раскомпенсации их полей: 


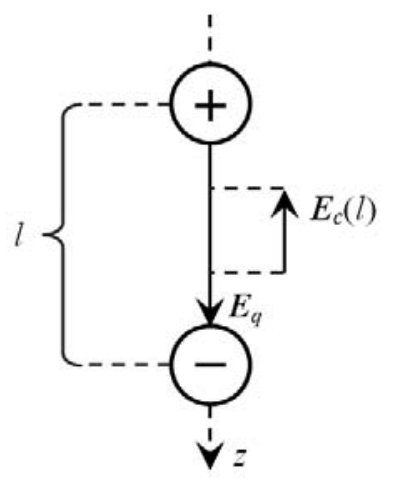

Рис. 3. Направление векторов $\boldsymbol{E}_{q}$ и $\boldsymbol{E}_{c}$ при раскомпенсации / компенсации полей зарядов диполя.

$$
F(l) \sim E_{q}-E_{c}(l)
$$

где $E_{q}-$ напряжённость электрического поля заряда, $E_{c}(l)$ напряженность скомпенсированной части электрического поля заряда (компенсация), причём $l \uparrow \Rightarrow E_{c} \downarrow \Rightarrow F \uparrow\left(l \downarrow \Rightarrow E_{c} \uparrow \Rightarrow F \downarrow\right)$. Также формулу для дипольной силы можно представить в следующем виде:

$$
F(l) \sim E_{d c}(l),
$$

где $E_{d c}(l)=E_{q}-E_{c}(l)$ - напряжённость нескомпенсированной части электрического поля заряда (раскомпенсация). Тут $l \uparrow \Rightarrow E_{d c} \uparrow \Rightarrow F \uparrow$ $\left(l \downarrow \Rightarrow E_{d c} \downarrow \Rightarrow F \downarrow\right)$. В этой формуле отсутствует проблема сингулярности в нулевой точке, так как:

$$
\begin{gathered}
l \rightarrow 0 \Rightarrow E_{d c} \rightarrow 0 \Rightarrow F \rightarrow 0 . \\
\left(E_{c} \rightarrow E_{q}\right)
\end{gathered}
$$

Если внешнее поле $\boldsymbol{E}_{0}$ разводит заряды то, согласно формуле дипольной силы, они наоборот притягиваются друг к другу. Так силовое действие внешнего поля $\boldsymbol{E}_{0}$ и дипольной силы $\boldsymbol{F}$ прямо противоположны и дальнейший рост амплитуды $l_{0}$ колебаний диполя останавливается при достижении равновесия этих сил.

На рис. 4 изображены графики зависимости силы взаимодействия между разноименными зарядами от расстояния между ними. Осцилляция атома всегда происходит в начале первой зоны (жирный участок линии) до его ионизации. Ядро остаётся внутри электронной оболочки и раскомпенсация полей разноименных зарядов во внешнем поле частичная. При бо́льших внешних полях начинает проявляться нелинейность (тонкий участок линии), а при дальнейшем их росте - ионизация атома (вторая зона), то есть отрыв электронной оболочки от ядра. В третьей зоне электронная оболочка и ядро уже отделены в пространстве друг от друга. И тут работает закон Кулона.

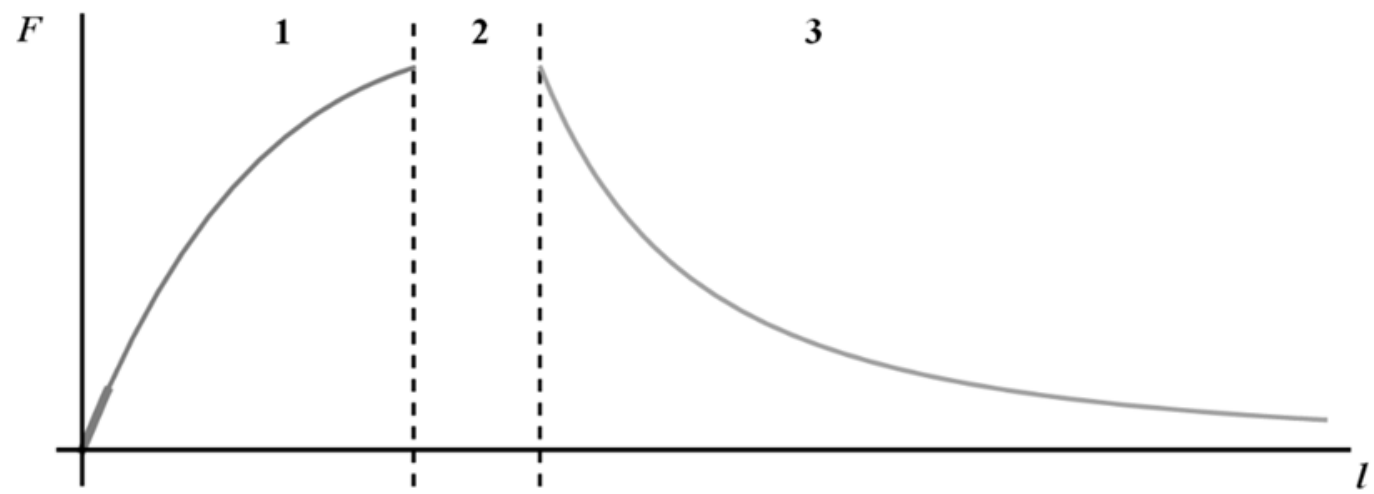

Рис. 4. Качественное изображение зависимости силы взаимодействия между зарядами $+q$ и $-q$ от расстояния между ними.

(1 - зона осцилляции, в которой работает формула дипольной силы (1), 2 - зона ионизации с отрывом электронной оболочки от ядра, 3 - зона действия закона Кулона между разделёнными зарядами, образованными в результате ионизации атома).

В нашей структурной атомной модели появляются внутридипольные квазистатические векторы $\boldsymbol{E}, \boldsymbol{H}$ и $\boldsymbol{S}$, что дает возможность рассмотреть в процессе осцилляции механизмы формирования потоков энергии: как реактивных $\boldsymbol{S}_{r}=\boldsymbol{E} \times \boldsymbol{H}$ (где $\boldsymbol{E}$ и $\boldsymbol{H}-$ компоненты электрической и магнитной напряженностей ближнего поля, которые имеют сдвиг фаз $\Delta \varphi \boldsymbol{E}, \boldsymbol{H}=\pi / 2$ ), так и активных $\boldsymbol{S}_{a}=\boldsymbol{E} \times \boldsymbol{H}$ (где $\boldsymbol{E}$ и $\boldsymbol{H}$ - компоненты электрической и магнитной напряженностей активного поля излучения со сдвигом фаз $\Delta \varphi \boldsymbol{E}, \boldsymbol{H}=0)$. Вместе с тем, как уже стало известно, $\boldsymbol{E} \equiv \boldsymbol{E}_{d c}$ и соответственно $\boldsymbol{H} \equiv \boldsymbol{H}_{d c}$ (где $\boldsymbol{H}_{d c}-$ напряжённость нескомпенсированной части магнитного поля движущегося заряда).

Итак, ближнее поле - это поле раскомпенсации. Оно появляется и усиливается, когда разноименные заряды во внешнем индуцирующем поле расходятся. И исчезает, когда они проходят через нулевую точку, то есть совмещаются и полностью компенсируют при интерференции силовое влияние полей друг друга (сами собственные поля всегда неотделимы от своих зарядов). При расхождении зарядов полная компенсация нарушается и начинает увеличиваться нескомпенсированное ближнее поле. При схождении, наоборот, 
интерферирующие поля разноименных зарядов совмещаются вплоть до полной компенсации внешних полей при их совмещении в нулевой точке. Таким образом, поля обеих зарядов являются «интерференционной маской» друг для друга, связывая изменяющуюся в процессе осцилляции часть силовых линий.

\section{ДИНАМИКА ПРОЦЕССОВ ОСЦИЛЛЯЦИИ В ДИПОЛЕ}

Рассмотрим процессы, протекающие при осцилляции диполя в 4-х фазах (четвертях периода). Соответствие между значениями $i, \boldsymbol{H}$, а также $\boldsymbol{E}_{d c}, \boldsymbol{F}$, как функций расстояния $l$ между разноименными зарядами диполя, и направлениями реактивного $\boldsymbol{S}_{r}$ и активного $\boldsymbol{S}_{a}$ потоков энергии в 4 -х фазах периода осцилляции показано на рис. 5.
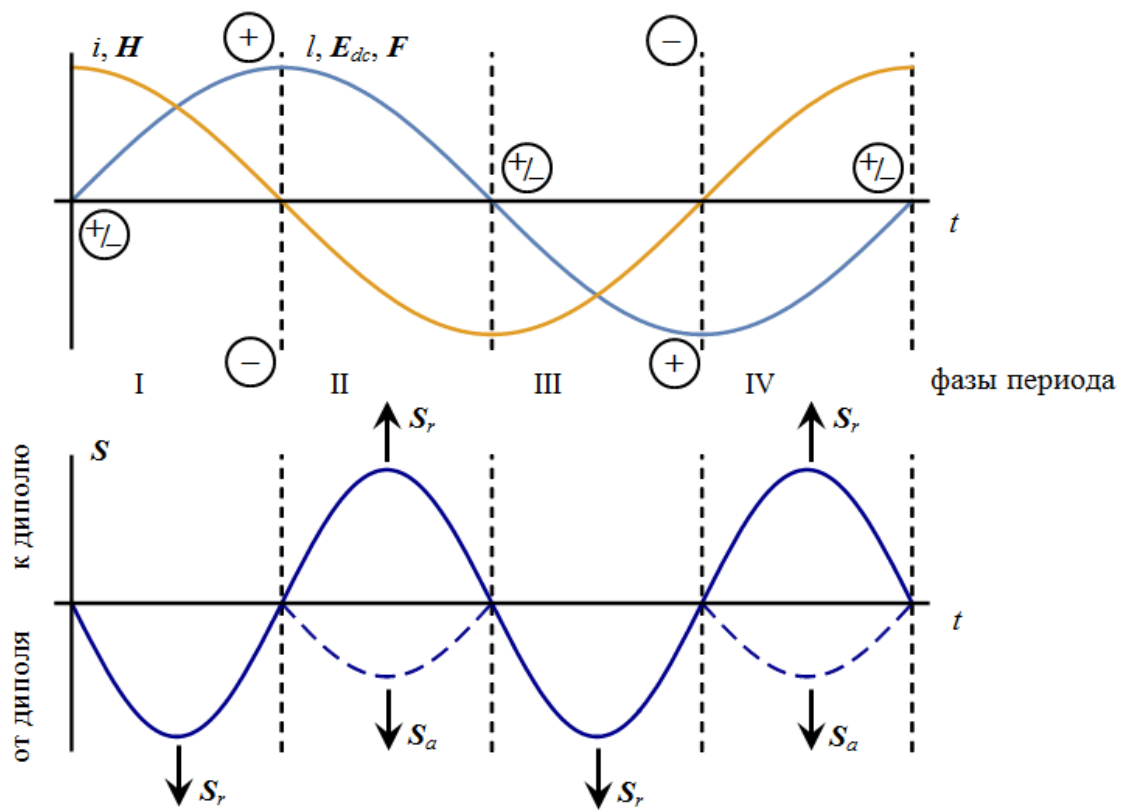

Рис. 5. Соответствие между значениями $i, \boldsymbol{H}$, а также $\boldsymbol{E}_{d c}, \boldsymbol{F}$, как функций плеча диполя $l$, и направлениями реактивного $\boldsymbol{S}_{r}$ и активного $\boldsymbol{S}_{a}$ потоков энергии в 4 -х фазах периода осцилляции.

В начале фазы I (рис. 6) модуль напряженности внешнего поля $E_{0}$ из нулевого значения начинает увеличиваться, а ее вектор направлен вверх. Из-за чего заряды из нулевой точки расходятся, и возникает ближнее поле. На этапе увеличения раскомпенсации возрастает плотность силовых линий. Квазистатическое ближнее поле распространяется со скоростью света, но концы силовых линий, плотность которых растёт пропорционально растущей напряжённости поля, замкнуты на заряды.

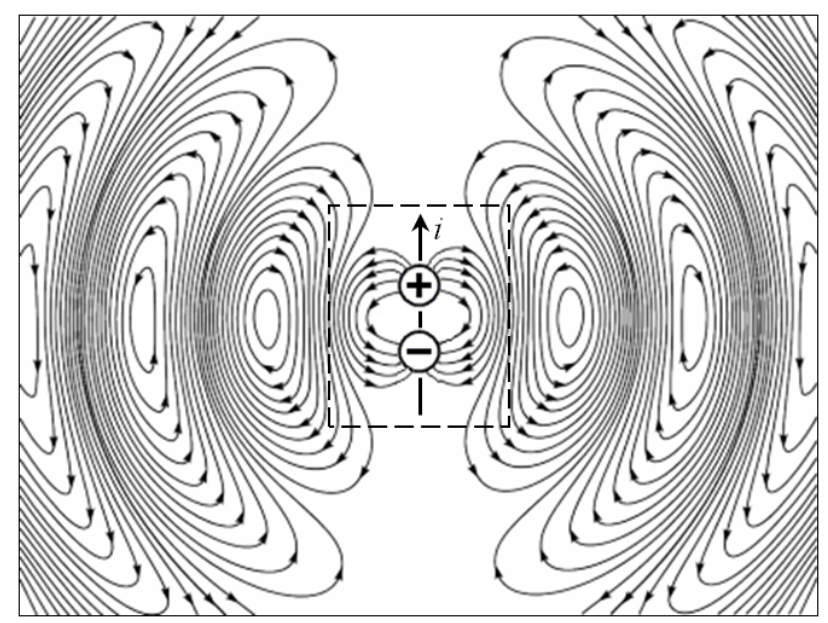

Рис. 6. Начало фазы I (рассматриваемая область обведена пунктирной линией).

Заряды из нулевой точки начали расходиться: $-q$ движется вниз, а ток направлен вверх.

Поток энергии $\boldsymbol{S}_{r}$ направлен от диполя вправо ортогонально его оси (рис. 7). Таким образом, при расхождении зарядов идёт накачка квазистатической энергии реактивного ближнего поля, силовые линии которого замкнуты на заряды. 


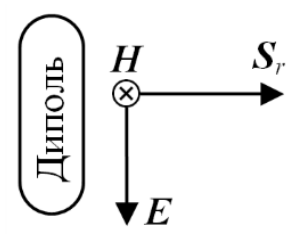

Рис. 7. Направление векторов $\boldsymbol{E}, \boldsymbol{H}$ и $\boldsymbol{S}_{r}$ в начале фазы I.

Когда заряды на мгновение останавливаются на максимуме $l$ (когда $l=l_{0}$ ), то $\boldsymbol{E}$ становится максимальной, а ток и $\boldsymbol{H}$ равны нулю (рис. 8). При прохождении нулевой точки ситуация обратная: $\boldsymbol{E}$ равна нулю, а ток и $\boldsymbol{H}-$ максимальны. Таким образом получается сдвиг фаз $\Delta \varphi_{E, \boldsymbol{H}}=\pi / 2$ и квазистатическое ближнее поле имеет чисто реактивный характер.

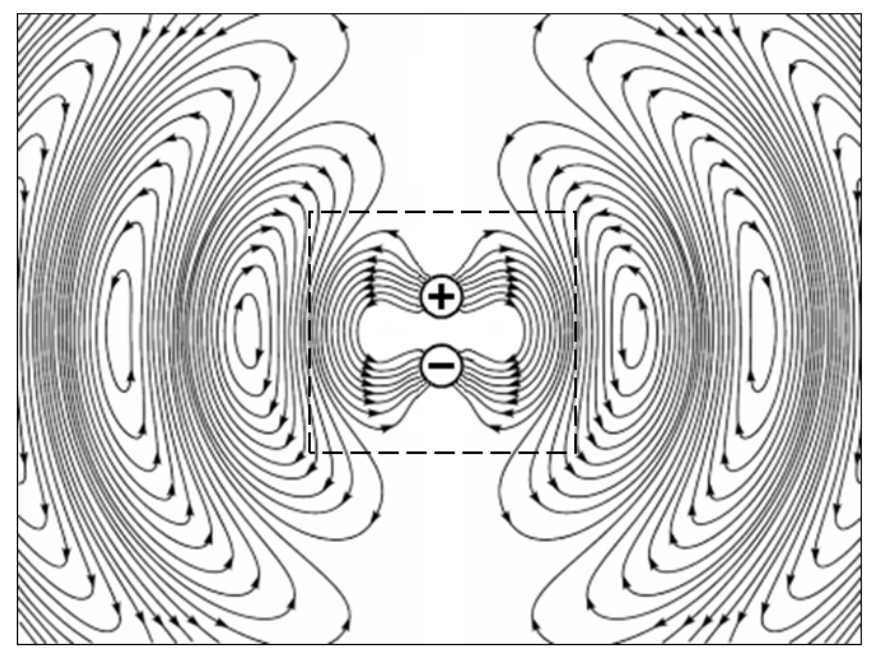

Рис. 8. Конец фазы I (рассматриваемая область обведена пунктирной линией).

Заряды разошлись на максимум $l_{0}$, ток отсутствует. Накачан максимум плотности силовых линий (энергии) ближнего поля. Все силовые линии все еще замкнуты на зарядах, а таким образом поля излучения пока нет

В начале фазы II (рис. 9) заряды сходятся: -q движется вверх, а ток направлен вниз. И поскольку взаимная компенсация увеличивается $\left(E_{c} \uparrow\right)$, то силовое поле уменьшается $\left(E_{d c} \downarrow\right)$ и таким образом часть силовых линий накачанного в фазе I ближнего поля оказываются избыточными. Они отрываются от разноименных зарядов, превращаясь в самозамкнутые петли поля излучения. Таким образом, процесс самозамыкания и преобразования ближнего поля в поле излучения идёт не в одной нулевой точке, где $E_{d c}=0$, а в течение всей фазы схождения зарядов за счёт процесса компенсации при уменьшении плеча диполя $l$. Идёт сброс избыточных силовых линий, которые самозакольцовываются и превращаются в замкнутые петли поля излучения.

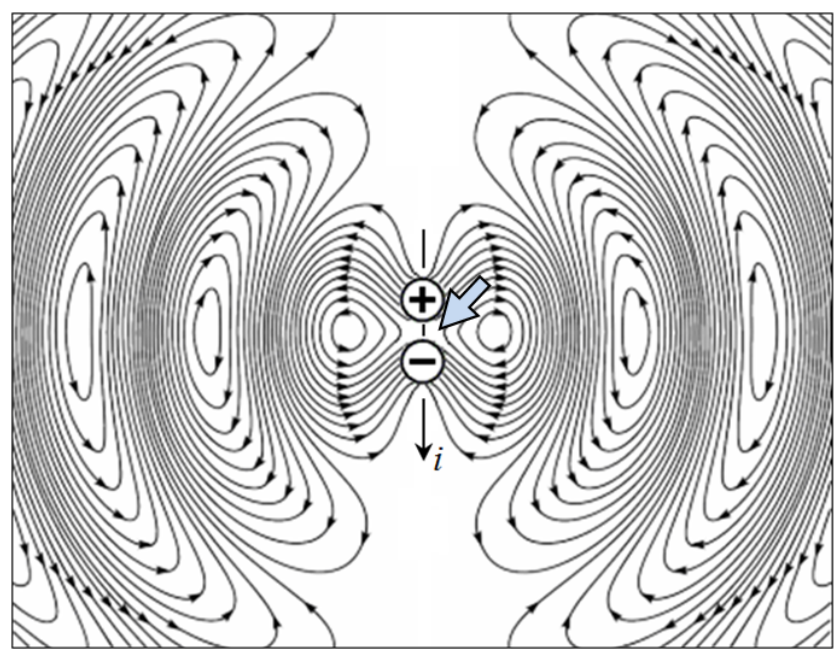

Рис. 9. Начало фазы II.

Заряды сходятся, и уменьшается $E_{d c}$ в процессе компенсации. Область самозакольцовывания обозначена наклонной стрелкой. 
В фазе II одновременно существуют два поля: уменьшающееся квазистатическое реактивное ближнее поле, которое замкнуто на заряды, и увеличивающееся активное свободное поле излучения (дальнее поле), оторвавшееся от зарядов и образовавшее самозамкнутые петли.

Рассмотрим направления потоков энергии этих двух полей. Реактивное ближнее поле фазы II показано на рис. 10. Вектор $\boldsymbol{E}$ и ток направлены вниз, поэтому вектор $\boldsymbol{H}$ направлен «к нам». Таким образом, вектор Пойнтинга реактивной энергии $\boldsymbol{S}_{r}$ направлен к диполю (ортогонально его оси).

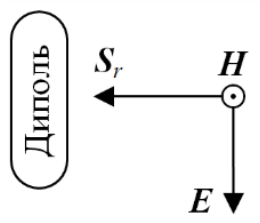

Рис. 10. Направление векторов $\boldsymbol{E}, \boldsymbol{H}$ и $\boldsymbol{S}_{r}$ в экваториальной плоскости диполя в фазе II.

Рассмотрим формирование активного поля излучения в фазе II. Поскольку при схождении зарядов их силовое поле уменьшается за счёт эффекта компенсации, то должна уменьшаться и плотность силовых линий замкнутых на заряды. Вектор $\boldsymbol{E}$ диполя направлен вниз и вблизи нулевой точки почти равен нулю при почти максимальном значении $\boldsymbol{H}$ (рис. 10). Квазистатическое ближнее поле, замкнутое на заряды, имеет чисто реактивный характер. После отрыва от зарядов и самозакольцовывания в петлю, $\boldsymbol{E}$ инвертируется (меняет направление на противоположное) и становится большим по величине. Это означает, что исчезает реактивный сдвиг по фазе $\Delta \varphi_{E, \boldsymbol{H}}=\pi / 2$ и данное реактивное поле превращается в активное поле излучения $\boldsymbol{S}_{a}\left(\Delta \varphi_{E, \boldsymbol{H}}=0\right)$ (рис. 11).

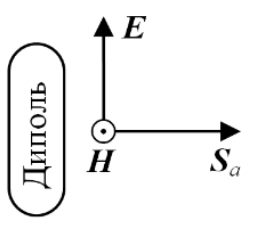

Рис. 11. Направление векторов $\boldsymbol{E}, \boldsymbol{H}$ и $\boldsymbol{S}_{a}$ в экваториальной плоскости диполя в фазе II.

Вектор $\boldsymbol{S}_{a}$ поля излучения направлен от диполя (ортогонально его оси) вдаль и растёт по величине по мере сокращения силовых линий ближнего поля, замкнутого на заряды. Примечательно, что в одно и то же время, в одном и том же месте существует два потока энергии: реактивный и активный. Причём они направлены в противоположные стороны. И реактивный уменьшается, а активный растёт так, что часть большой реактивной энергии трансформируется в малую активную (рис. 5,12 ). При схождении диполь работает как преобразователь накопленной в предыдущей фазе энергии ближнего поля в виде пучка силовых линий связанных с зарядами в замкнутые петли поля излучения. Таким образом, преобразователем полей является диполь.

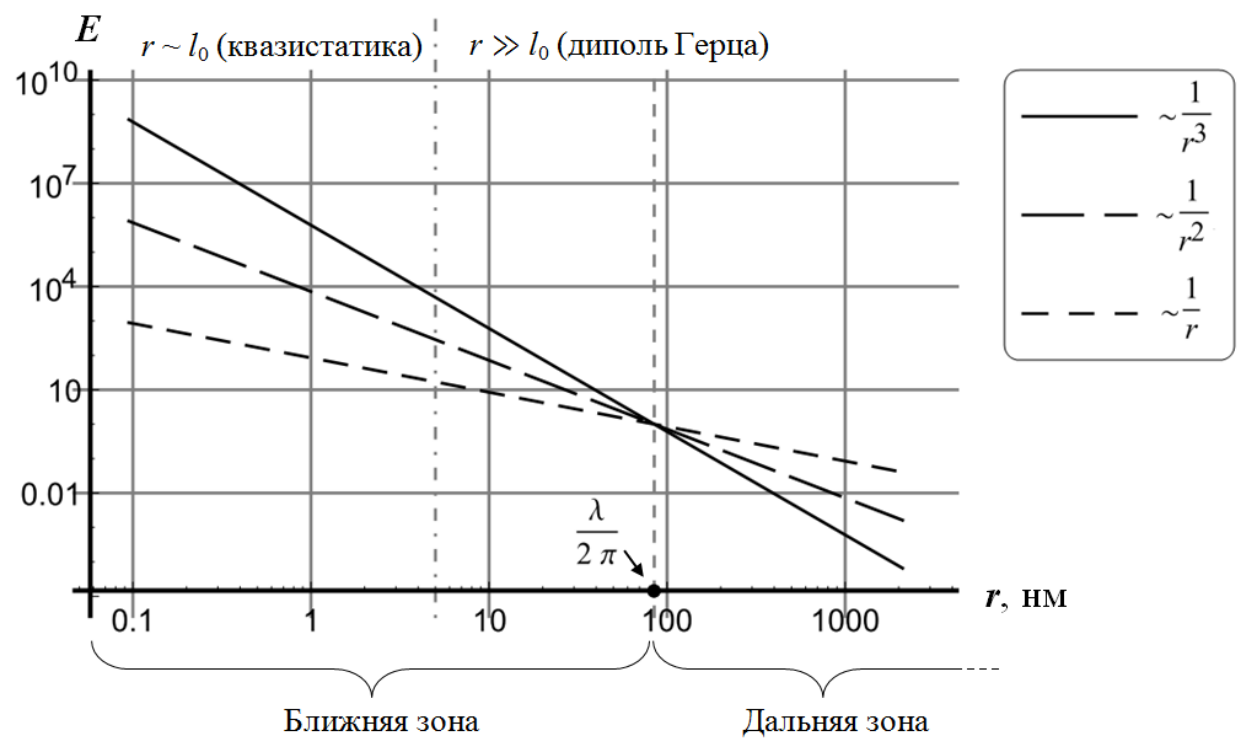

Рис. 12. Графики зависимости относительных значений напряженности электрического поля от расстояния от диполя для 3-х компонент электрического поля $(\lambda=532$ нм$)$. 
Поле излучения рождается непосредственно в точке самозакольцовывания, так как направление вектора $\boldsymbol{E}$, а таким образом и потока энергии $\boldsymbol{S}$, меняется на противоположное в момент отрыва избыточной силовой линии от зарядов и её самозакольцовывания в замкнутую петлю поля излучения (рис. 11).

Рассмотрим соотношение этих потоков энергии $\left(\boldsymbol{S}_{\mathrm{r}}\right.$ и $\left.\boldsymbol{S}_{a}\right)$. Все осцилляторы являются своего рода колебательным контуром с реактивными и активными потоками энергии, причём реактивные гораздо больше активных - в $Q$ раз (где $Q$ - добротность контура). При включении внешнего поля амплитуда реактивных колебаний начинает расти и этот рост ограничивается только наличием активных потерь, выводящих энергию из осциллятора. В колебательном контуре это активное сопротивление потерь, а в диполе - это активные потери на излучение энергии $\boldsymbol{S}_{a}$. Как видно из рис. 12 , в локальной зоне диполя (при $r \sim l_{0}$ ) амплитуды реактивных полей на порядки превышают амплитуды активных полей излучения. Аналогичная ситуация и в радиотехнике: на резонансе реактивные поля (напряженность $\boldsymbol{E}$ на конденсаторе и ток, а таким образом и $\boldsymbol{H}$ катушки индуктивности) возрастают в $Q$ раз. Аналогично и в диполе $\boldsymbol{S}_{r} \gg \boldsymbol{S}_{a}$. Это означает, что в фазе схождения, когда одновременно существуют два поля с противоположными направлениями потоков энергии, активный поток поля излучения $\boldsymbol{S}_{a}$ незначителен по сравнению с реактивным $\boldsymbol{S}_{r}$. Поэтому плотность оторвавшихся и самозамкнувшихся петель поля излучения много меньше плотности замкнутых на заряды силовых линий ближнего поля.

В конце фазы II (рис. 13) силовые линии ближнего поля, оторвавшись от своих зарядов, самозамкнулись в петли поля излучения. Абсолютные значения тока и $\boldsymbol{H}$ в нулевой точке максимальны, но $\boldsymbol{E}_{d c}=0$ и реактивного потока $\boldsymbol{S}_{r}$ нет. Справа от диполя (рис. 11$)$ вектор $\boldsymbol{H}$ направлен «к нам», вектор $\boldsymbol{E}$ поля излучения направлен вверх, следовательно, максимальный активный поток $\boldsymbol{S}_{a}$ направлен от диполя (ортогонально его оси). Силовые линии накачанного в предыдущей фазе ближнего поля преобразованы диполем в петли свободного поля излучения (рис. 11,13$)$. А реактивный поток $\boldsymbol{S}_{r}$ равен нулю, поскольку электрическое поле зарядов в нулевой точке полностью скомпенсировано.

Далее всё повторяется во втором полупериоде, только с инверсией знаков зарядов и токов - формируются силовые линии следующего уже инверсного полупериода поля излучения. И самозакольцованные петли активного поля распространяются от диполя (ортогонально его оси) влево.

Итак, отрыв силовых линий с их самозамыканием в петли поля излучения идёт только в фазах II и IV схождения зарядов и уменьшения величины раскомпенсации (рис. 5). Происходит преобразование ближнего квазистатического поля, накачанного в фазах I и III расхождения, в свободное поле излучения, распространяющееся из ближней зоны в дальнюю.

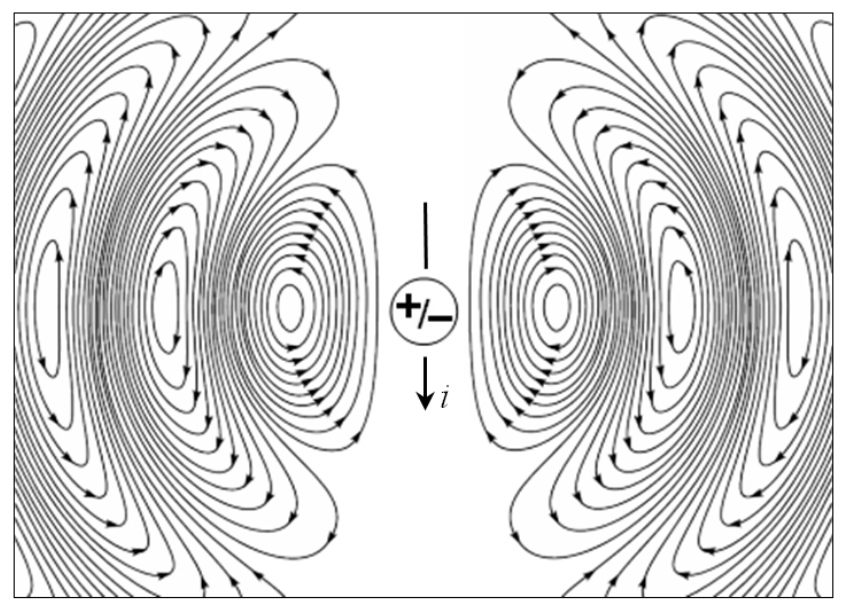

Рис. 13. Конец фазы II.

Заряды находятся в нулевой точке и их поля, а таким образом и силовые линии реактивного $\boldsymbol{S}_{r}$ ближнего поля, полностью скомпенсировались интерференцией. Осталось только небольшое количество оторвавшихся и самозакольцевавшихся петель распространяющегося в дальнюю зону активного $\boldsymbol{S}_{a}$ поля излучения.

В фазах расхождения (I и III) за счёт раскомпенсации полей увеличивается плотность силовых линий реактивного ближнего поля, замкнутых на заряды. Нарастает энергия ближнего поля, но не происходит процесс преобразования его в замкнутые петли свободного поля излучения. Реактивный вектор Пойнтинга $\boldsymbol{S}_{r}$ в нарастающем ближнем поле направлен от диполя (ортогонально его оси).

В фазах схождения (II и IV) за счёт компенсации полей уменьшается плотность силовых линий реактивного ближнего поля, замкнутых на заряды. Реактивный вектор Пойнтинга $\boldsymbol{S}_{r}$ направлен к диполю (ортогонально его оси). Сокращающиеся силовые линии самозакольцовываются и образуют замкнутые петли поля излучения. В самозамкнутых петлях направление вектора $\boldsymbol{E}$, а следовательно и потока энергии в момент отрыва избыточных силовых линии от зарядов, меняется на противоположное (с $\boldsymbol{S}_{r}$ на $\left.\boldsymbol{S}_{a}\right)($ рис. $5,10-11)$. В этот 
момент изменяется и сдвиг фаз с реактивного $\Delta \varphi_{\boldsymbol{E}, \boldsymbol{H}}=\pi / 2$ на активный $\Delta \varphi_{\boldsymbol{E}, \boldsymbol{H}}=0$, поскольку поле $\boldsymbol{E}$ из стремящегося к нулю $\boldsymbol{E}_{d c}$ становится большим и синфазным с большим $\boldsymbol{H}$ в нулевой точке.

Таким образом, на этапе схождения имеем одновременно два типа полей: реактивное и активное, потоки энергии которых $\boldsymbol{S}_{r}$ и $\boldsymbol{S}_{a}$ направлены в противоположные стороны (рис. 5). Это сокращающееся ближнее поле, замкнутое на заряды, и свободное от зарядов растущее поле излучения в виде самозамкнувшихся петель. Соотношение этих полей непрерывно меняется вплоть до нулевой точки, в которой $\boldsymbol{E}_{d c}=0$. Но остаются замкнутые петли свободного поля излучения, распространяющиеся в дальнюю зону (рис. 13).

Итого имеем следующую ситуацию. В фазе I поток энергии $\boldsymbol{S}_{r}$ направлен от диполя и накачивает энергию ближнего поля. В фазе II $\boldsymbol{S}_{r}$ направлен к диполю. В фазе III поток энергии, но уже инвертированный, снова направлен от диполя и накачивает энергию ближнего поля противоположной полярности. В фазе IV поток реактивной энергии снова направлен к диполю. Таким образом, поток реактивной энергии ближнего поля 4 раза за период меняет направление своего движения. И поэтому в среднем за период реактивный поток ближнего поля равен нулю.

В литературе соотношение амплитуд реактивных и активных полей обычно приводятся на графиках в линейном или полулогарифмическом масштабе [1]. Но соотношение амплитуд в ближней зоне сложно сосчитать по крутым гиперболическим кривым. Поэтому данные графики удобнее отображать в логарифмическом масштабе (рис. 12). На графики квазистатических полей ограничение диполя Герца $r \gg l_{0}$ не распространяется, так как силовые линии начинаются непосредственно от своих источников - зарядов и их движения (токов). Поля в локальной зоне на расстоянии $r \sim l_{0}(\sim 1 \AA)$ становятся более сложными. К ним добавляются мультиполя разных порядков и их расчёт резко усложняется. И поэтому имеет смысл проведенный на уровне классической электродинамики качественный анализ физических механизмов формирования потоков энергии ближних и дальних полей осциллирующего диполя, основанный на использовании квазистатических силовых линий их электрических зарядовых полей $\boldsymbol{E}$ (полей Кулона) и магнитных полей $\boldsymbol{H}$ токов (полей Био-Савара) для анализа потоков энергии: как реактивных $\boldsymbol{S}_{r}$ при $\Delta \varphi_{\boldsymbol{E}, \boldsymbol{H}}=\pi / 2$, так и активных $\boldsymbol{S}_{a}$ при $\Delta \varphi_{\boldsymbol{E}, \boldsymbol{H}}=0$.

\section{МЕХАНИЗМ ДВИЖЕНИЯ ВОЛНЫ ПОЛЯ ИЗЛУЧЕНИЯ}

Приведем два уравнения Максвелла (закон Ампера-Максвелла и закон индукции Фарадея) для вакуума (в системе СИ):

$$
\begin{aligned}
\operatorname{rot} \boldsymbol{H} & =\boldsymbol{j}+\varepsilon_{0} \frac{\partial \boldsymbol{E}}{\partial t}, \\
\operatorname{rot} \boldsymbol{E} & =-\mu_{0} \frac{\partial \boldsymbol{H}}{\partial t} .
\end{aligned}
$$

Чтобы выяснить механизм распространяется электромагнитной волны, выберем начальную точку при максимальных значениях $\boldsymbol{E}$ и $\boldsymbol{H}$ (рис. 14). Это определяет поведение вектора $\boldsymbol{E}$ в следующий момент времени его модуль может только уменьшаться.

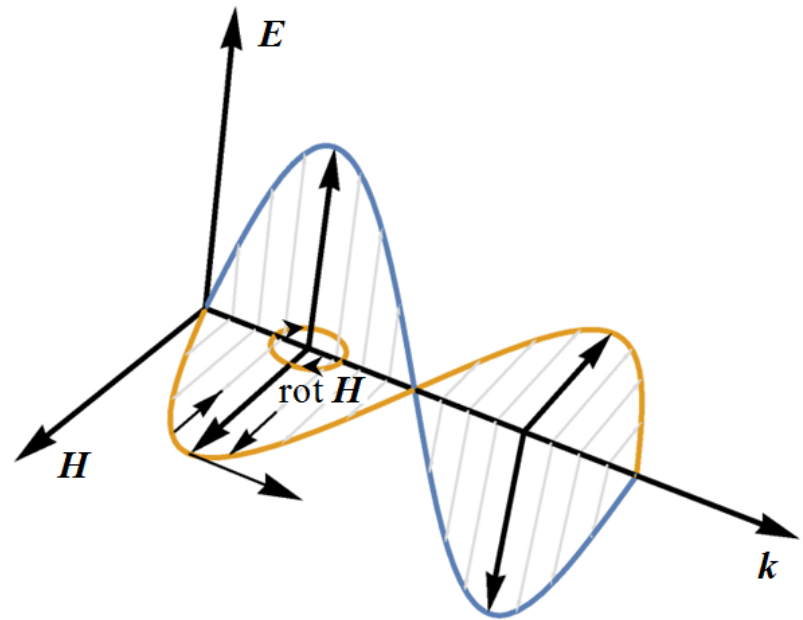

$\operatorname{rot} \boldsymbol{H} \in$ пл. $(\boldsymbol{H}, \boldsymbol{k}), \quad \operatorname{rot} \boldsymbol{H} \perp \boldsymbol{E}$

a

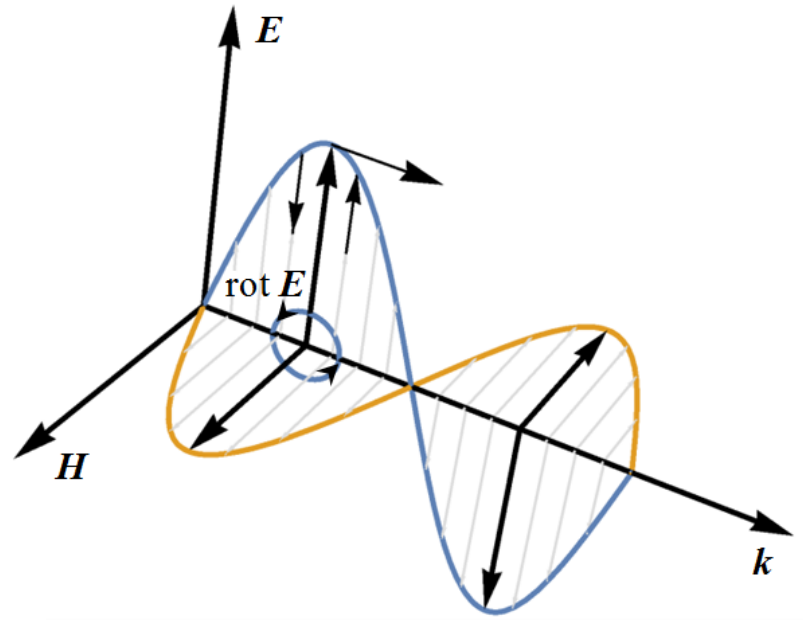

$\operatorname{rot} \boldsymbol{E} \in$ пл. $(\boldsymbol{E}, \boldsymbol{k}), \quad \operatorname{rot} \boldsymbol{E} \perp \boldsymbol{H}$

Рис. 14. Векторное представление активной электромагнитной волны поля излучения с изображением вихря $\boldsymbol{H}$ (а) и вихря $\boldsymbol{E}($ б).

Амплитуды $\boldsymbol{E}$ и $\boldsymbol{H}$ синфазны $\left(\Delta \varphi_{\boldsymbol{E}, \boldsymbol{H}}=0\right)$. 
Уменьшение $\boldsymbol{E}$, согласно уравнению (2), вызовет $\operatorname{rot} \boldsymbol{H}$. Вихрь магнитного поля в этой точке лежит в плоскости $(\boldsymbol{H}, \boldsymbol{k})$ (рис. 14, а). Магнитное поле вихря суммируется со значением $\boldsymbol{H}$ переднего фронта и вычитается из заднего фронта. Следовательно, максимум $\boldsymbol{H}$ смещается вперёд (в направлении $\boldsymbol{k}$ ), а, таким образом, в исходной точке $\boldsymbol{H}$ уменьшается.

В соответствии с уравнением (3), уменьшение $\boldsymbol{H}$ в исходной точке вызовет в ортогональной плоскости $(\boldsymbol{E}, \boldsymbol{k})$ вихрь $\boldsymbol{E}$ (рис. 14, б), значение которого будет суммироваться с передним фронтом $\boldsymbol{E}$ и вычитаться из заднего. Максимум $\boldsymbol{E}$ сместится вперёд (в направлении $\boldsymbol{k}$ ), а в сходной точке $\boldsymbol{E}$ уменьшится. Это опять вызовет такой вихрь $\boldsymbol{H}$, который снова переместит максимум $\boldsymbol{H}$ вперёд, и так далее.

Таким образом, вся волна путём взаимного перелива своих гребней будет распространяться вперёд (в направлении $\boldsymbol{k}$ ).

Волна не есть распространяющиеся колебания, как ошибочно иногда говорят [10]. Любое электромагнитное поле можно разложить на активные и реактивные составляющие. В колебаниях $\Delta \varphi_{E, \boldsymbol{H}}=\pi / 2$ и поэтому когда амплитуда одного из полей максимальна, амплитуда второго равна нулю и наоборот. А в волнах $\Delta \varphi_{E, \boldsymbol{H}}=0$ - амплитуды синфазны. Значит, энергии реактивных полей полностью перекачиваются друг в друга во всей области колебаний. Этого не происходит в волнах, в которых амплитуды $\boldsymbol{E}$ и $\boldsymbol{H}$ никогда не обнуляются - они просто переливают гребни друг друга в направлении распространения $(\boldsymbol{k})$.

\section{ВЫВОДЫ}

Показано, что попытка применения закона Кулона к атомному диполю должна привести к сингулярности в нулевой точке и невозможности поляризации атома в дальнейшем. Предложена модель диполя со структурой атома, которая более приближена к реальности, чем модель диполя Герца или стандартная модель с точечными зарядами.

Классическая модель диполя с точечными зарядами к атомному диполю неприменима, поскольку положительный заряд ядра всегда находится внутри распределенного отрицательного заряда электронной оболочки и всегда имеет место то или иное значение компенсации полей этих зарядов.

Главный недостаток стандартной модели диполя с точечными зарядами состоит в постоянном значении суперпозиции силовых линий полей зарядов диполя в процессе его осцилляции, что принципиально не позволяет объяснить физический механизм преобразования ближнего поля в поле излучения.

Показано, что при осцилляции электронной оболочки под действием внешнего поля происходит частичная раскомпенсация полей зарядов, которая зависит от плеча диполя $l$ и определяет силу притяжения разноимённых зарядов.

Предложена функциональная формула дипольной силы, возникающей между разноименными зарядами в процессе осцилляции диполя и раскомпенсации их полей: $F(l) \sim E_{d c}(l)$.

Введено понятие локальной зоны диполя. Это зона $r \sim l_{0}$, в которой диполь уже не бесконечно малый элемент тока, как в диполе Герца, а структурный элемент, в котором различаются разноименные заряды, генерирующие электрические $\boldsymbol{E}$ и магнитные $\boldsymbol{H}$ поля, а также реактивные $\boldsymbol{S}_{r}$ и активные $\boldsymbol{S}_{a}$ потоки энергии. Поля в локальной зоне на расстоянии $r \sim l_{0}(\sim 1 \AA)$ становятся более сложными. К ним добавляются мультиполя разных порядков, их расчёт резко усложняется и поэтому имеет смысл проведенный на уровне классической электродинамики качественный анализ физических механизмов формирования потоков энергии ближних и дальних полей осциллирующего диполя, основанный на использовании квазистатических силовых линий их электрических зарядовых полей $\boldsymbol{E}$ (полей Кулона) и магнитных полей $\boldsymbol{H}$ движущихся зарядов (полей БиоСавара) для анализа потоков энергии: как реактивных $\boldsymbol{S}_{r}$ при $\Delta \varphi_{\boldsymbol{E}, \boldsymbol{H}}=\pi / 2$, так и активных $\boldsymbol{S}_{a}$ при $\Delta \varphi_{\boldsymbol{E}, \boldsymbol{H}}=0$.

Применение в анализе квазистатических силовых линий, начинающихся непосредственно от их источников - зарядов и токов, позволило в предложенной модели диполя снять ограничения диполя Герца $r \gg l_{0}$ и дать физический анализ механизмов формирования реактивных ближних полей и трансформации их в активные поля излучения, а также описать потоки энергии в локальной зоне осциллятора $r \sim l_{0}$.

Показано, что в фазах схождения в момент отрыва силовых линий ближнего поля от зарядов и их самозакольцовывания в петли свободного поля излучения направление вектора $\boldsymbol{E}$, а таким образом и потока энергии, меняется на противоположное (с $\boldsymbol{S}_{r}$ на $\left.\boldsymbol{S}_{a}\right)$. Так в момент отрыва избыточных силовых линий от зарядов поле излучения рождается непосредственно в точке самозакольцовывания в замкнутую петлю силовых линий.

Отрыв силовых линий с их самозамыканием идёт только на этапах схождения зарядов и уменьшения величины раскомпенсации. Идёт преобразование ранее накачанной энергии ближнего квазистатического поля в энергию свободного поля излучения, распространяющегося из ближней зоны в дальнюю.

Описан механизм движения волны поля излучения путём перелива гребня в соответствии с уравнениями Максвелла. Показано принципиальное отличие волны (активного поля с $\Delta \varphi_{E, \boldsymbol{H}}=0$ ) от колебания (реактивного поля с $\left.\Delta \varphi_{\boldsymbol{E}, \boldsymbol{H}}=\pi / 2\right)$.

\section{БЛАГОДАРНОСТЬ}

Авторы выражают благодарность Государственному фонду фундаментальных исследований Украины (грант Ф76/33332). 


\section{СПИСОК ЛИТЕРАТУРЫ}

1. Sydorenko V.S., Gayday Y.O., Zhyla S.V., Sinkevych O.V. Distribution of the Poynting vector in the near field of the Hertz dipole // Bullet. Univ. Kiev. Ser.: Radiophys. and Electron. - 2003. - Iss. 1. - P. 55-59. (in Ukrainian)

2. Sydorenko V.S., Gayday Y.O., Zhyla S.V. Peculiarity of near-field of Hertz dipole // Bullet. Univ. Kiev. Ser.: Phys. \& Math. 2005. - Iss. 2. - P. 365-372. (in Ukrainian)

3. McDonald K.T. Radiation in the Near Zone of a Hertzian Dipole [Electronic resource] // Joseph Henry Laboratories, Princeton University. - 2004. - URL: http://www.physics.princeton.edu/ mcdonald/examples/nearzone.pdf

4. McDonald K.T. Flow of Energy and Momentum in the Near Zone of a Hertzian Dipole [Electronic resource] // Joseph Henry Laboratories, Princeton University. - 2014. - URL:

http://www.physics.princeton.edu/ mcdonald/examples/hertzian momentum.pdf

5. Staliunas K., Markoš P., Kuzmiak V. Scattering properties of a PT dipole // Phys. Rev. A. - 2017. - Vol. 96. -Iss. 4. - P. 043852.

6. Wong H.M.K., Dezfouli M.K., Axelrod S., Hughes S., Helmy A.S. Theory of hyperbolic stratified nanostructures for surface enhanced Raman scattering // Phys. Rev. B. - 2017. - Vol. 96. - P. 205112.

7. Cao D., Cazé A., Calabrese M., Pierrat R., Bardou N., Collin S., Carminati R., Krachmalnicoff V., De Wilde Y. Mapping the radiative and the apparent non-radiative local density of states in the near field of a metallic nanoantenna // ACS Photonics. 2015. - Vol. 2. - Iss. 2. - P. 189-193.

8. Boutelle R.C., Yi X., Neuhauser D., Weiss S. SOFI for Plasmonics: Extracting Near-field Intensity in the Far-Field at High Density // ACS Nano. - 2016. - Vol. 10. - Iss. 8. - P. 7955-7962.

9. Landau L.D., Lifshitz E.M. Course of Theoretical Physics. Vol. 2: The Classical Theory of Fields (8th ed.). - Moscow: Fizmatlit, 2012. - 536 p. (in Russian)

10. Kuznetsov S.I. Oscillations and waves. Geometric and wave optics (2nd ed.). - Tomsk: TPU, 2007. - 170 p. (in Russian)

11. Stas D.V., Plyusnin V.F. Quantum Mechanics of Molecules. Part 1: Atom. - Novosibirsk: NSU, 2008. - 186 p. (in Russian) 